\title{
What is information science for? A review of Search foundations
}

\author{
Tim Gorichanaz \\ Drexel University, Philadelphia, PA, USA \\ E-mail: gorichanaz@drexel.edu
}

Sachi Arafat and Elham Ashoori. Search Foundations: Toward a Science of Technology-Mediated Experience. Cambridge, MA: MIT Press, 2019. 448 pp. ISBN 9780262038591 Hardcover, \$65.00.

What is information science for? As technologies and cultures change, this is a question that keeps bubbling to the surface (Borko, 1968; Capurro, 1992; Gorichanaz, 2017). And along with it, related questions: What does it mean to make progress in this field? What is the relation between the various areas of the information field? Are these sciences? In this book, Arafat and Ashoori develop answers to these questions - and they offer the conceptual tools needed to keep answering them far into the future. This is an ambitious work with a broad scope, one that examines and challenges the foundations of the information field writ large.

In brief, the authors reimagine the information field as a science of technologymediated experience. Along the way, they develop in detail the concepts of technology, mediation and experience. Some contributions of the book are metatheoretical, while others are directly substantive.

First, I will discuss some of the metatheoretical contributions. The first four chapters of the book articulate the need for and importance of basic research in the field. Arafat and Ashoori start by observing the broadening scope and ubiquity of information technology today. They suggest this goes along with the trend in information science and retrieval ${ }^{1}$ (styled IR\&S) of moving from ad-hoc projects and research questions to non-ad-hoc ones. This movement demands conceptual thinking. As the authors write, "Technical questions on interface and algorithm design, or about the functionality and usability of a search interface, presuppose more basic questions"

\footnotetext{
${ }^{1}$ The authors use IR\&S as an umbrella term for both information science (IS) and information retrieval (IR). In general they are not concerned with differentiating these two fields - wisely, in my opinion, as the division may be specious to begin with. But puzzlingly they do seem to separate them at times, such as in the claim that "when the purpose of IR\&S is mentioned, it should be taken to refer to the purpose of IS, which employs IR to partially fulfill this purpose" (p. 225).
} 
(p. 66). Arafat and Ashoori make an extended case for the importance of such work, which they term "foundations" research, organized into three types of arguments (pp. 211-212):

- Pragmatic: Answering basic questions can resolve practical concerns about what to build and how to evaluate it in the modern context.

- Discourse: Foundations research is required to define what progress can mean in a given discourse, and to show that progress is being made.

- Logical: Just as houses need foundations, sciences need clear methods and concepts.

Arafat and Ashoori dedicate their fifth chapter to following through on the need for foundations work that they articulated in the first four chapters. Here we find the directly substantive contributions of the book. As the title of the book suggests, the authors focus on search as the central phenomenon of IR\&S. If one would object that this focus is too narrow, we find soon enough that search is broadly conceptualized as technology-mediated experience. Arafat and Ashoori describe four levels of phenomena in experience: the human level; the mediation level; the semantic level; and the interaction level. In this framework, they pose two key questions:

First, what are the available functions [for] entities... at each level: human acts/experience in general, mediation acts/experiences, semantic (meaning suggesting/generating) and interaction events? Second, as we are particularly interested in the mediation level, what are the characteristics shared by the phenomena therein by which this level could be characterized? (p. 239)

They answer these questions by way of Heidegger's discussion of the four types of causes (drawn in turn from Aristotle) in what amounts to an interesting and novel analysis of search: Information needs are the final and efficient causes; queries are the formal cause; and the system is the material cause. Finally, the document, on this account, is "what is brought forth by the means of the four causes" (p. 263). What all this means for IR\&S is:

If a retrieval system is supposed to provide access to documents as things, or to documents as experiences, then IR\&S has to first develop a way of talking about (not just representing) the relevant objects or "basic forms" of that thing or experience, respectively. (pp. 270-271)

This is a central challenge for the field moving forward.

This is a demanding work that requires patience and care. In part, this is due to the structure, which is deeply sectioned and heavily cross-referenced. For instance, on p. 255, we find the parenthetical instructions: "cf. Sections 1.1.3, 2.3, and 3.3.4." It may be that precise argumentation demands such complexity. Yet there are certainly places where redundancy could have been eliminated - e.g., "related through relations" (p. 220) and "stored inside a data store" (p. 264) - and one suspects that the text could have been edited down throughout, for clarity and conciseness. 
In addition, in places the discussion relies on secondary references to a troubling extent. For example, on p. 252, the authors give a definition of information need, from Cole (2012, p. 189), in a footnote that reads: "As quoted in in [sic] the review of Cole's book by Ford (Nigel, 2013, p. 2596)." And indeed, the reference is listed as "Nigel, Ford" rather than "Ford, Nigel." Lastly, what is perhaps more serious than the use of secondary literature and the lack of proofreading, the book is missing a few key sources that would have reshaped the discussion significantly had they been duly engaged. In the spirit of moving these discussions forward, I will give three examples:

- Bates (1999): In this classic paper, Bates articulates the concept of a metadiscipline, which operates and theorizes on the objects of other disciplines. She argues that information science is a metadiscipline to the extent that it researches and theorizes the documents created and circulated through other disciplines. Arafat and Ashoori do not seem to be aware of this concept, but it must be considered in any attempt to reformulate the science of information science. Indeed the authors seem to struggle to express what Bates has encapsulated in this term, as they see IR\&S as a "mixed scientific-humanistic discipline" (p. 345) that "has both a generic and specific nature" (p. 297) and is "lacking the types of theories common to natural science" (p. 183).

- Gorichanaz and Latham (2016): In this paper, Latham and I conceptualize the document as experience, inspired by Heidegger's phenomenology, as what reveals itself when a person and an object come together. Arafat and Ashoori likewise draw on Heidegger to make a similar argument. Puzzlingly, the authors do cite our work (albeit in a footnote, on p. 269), yet do not seem to realize that they are re-stating our argument.

- Hjørland (2010): In this paper, Hjørland conceptualizes relevance from the socio-cognitive perspective, arguing that a person's situation, task and goals impinge on a document's relevance. Arafat and Ashoori seem to reinvent Hjørland's wheel without apparent knowledge of this work.

I would like to stress that the authors should be forgiven for overlooking some works given the admirable breadth and scope of this book. Arafat and Ashoori offer much novelty, and much to build upon. Search Foundations is a worthy contribution, one that can be engaged with in all corners of IR\&S, by whatever name.

\section{References}

Bates, M. J. (1999). The invisible substrate of information science. Journal of the American Society for Information Science, 50(12), 1043-1050.

Borko, H. (1968). Information science: What is it? American Documentation, 19(1), 3-5.

Capurro, R. (1992). What is information science for? A philosophical reflection. In P. Vakkari \& B. Cronin (Eds.), Conceptions of library and information science. Historical, empirical and theoretical perspectives (pp. 82-98). London: Taylor Graham. 
Gorichanaz, T. (2017). Applied epistemology and understanding in information studies. Information Research, 22(4), paper 776. Retrieved from http://InformationR.net/ir/22-4/paper776.html

Gorichanaz, T., \& Latham, K. F. (2016). Document phenomenology: a framework for holistic analysis. Journal of Documentation, 72(6), 1114-1133.

Hjørland, B. (2010). The foundation of the concept of relevance. Journal of the American Society for Information Science and Technology, 61(2), 217-237. 